\title{
HPLC separation of panthenol enantiomers on different types of chiral stationary phases
}

\author{
Anna Lomenova, Katarína Hroboňová, Terézia Šolónyová \\ Slovak University of Technology in Bratislava, Faculty of Chemical and Food Technology, \\ Institute of Analytical Chemistry, Radlinského 9, 81237 Bratislava, Slovak Republic \\ katarina.hrobonova@stuba.sk
}

\begin{abstract}
Panthenol is a biologically active compound closely related to vitamin B5 (pantothenic acid). This work deals with the separation of panthenol enantiomers using high performance liquid chromatography. Different types of chiral stationary phases ( $\beta$-cyclodextrin, isopropyl carbamate cyclofructan 6 , amylose tris(3,5-dimethylphenylcarbamate)) were tested in normal phase separation mode. Effect of mobile phase composition on the resolution and retention of enantiomers was studied. Two types of detectors, low-wavelength UV and polarimetric, were used. The optimal chromatographic system includes a chiral stationary phase based on amylose and a mobile phase of hexane/ethanol $(60 / 40, \mathrm{v} / \mathrm{v})$ where the resolution of enantiomers reached the value $R_{\mathrm{s}}=2.49$. Suitable chromatographic conditions were applied for the determination of panthenol enantiomers in samples of pharmaceutical preparations with the obtained recovery of more than $92 \%$. Linearity of the high performance liquid chromatography method with spectrophotometric detection was from $1.0 \times 10^{-3}$ to $1.3 \mathrm{mg} \mathrm{mL}^{-1}\left(R^{2}=0.998\right)$, with the limit of detection of $0.3 \times 10^{-3} \mathrm{mg} \mathrm{mL}^{-1}$ for both enantiomers.
\end{abstract}

Key words: chiral stationary phase, HPLC, panthenol, pharmaceutical preparations, polarimetric detection

\section{Introduction}

Panthenol (Fig. 1) is a biologically active compound metabolising in living organism into panthotenic acid known as vitamin $\mathrm{B} 5$, which is a constituent of hair and skin. Lack of vitamin B5 causes hair depigmentation, skin irritation and dermatitis (Xie et al., 2009). Panthenol occurs in D-enantiomeric form (Dexpanthenol) which is biologically active, and in inactive L-enantiomeric form. In some cases, L-panthenol (3-[(2R)-(2,4-dihydroxy-3,3-dimethylbutanoyl)amino]propanoic acid) can block the effect of D-panthenol (3-[(2S)-(2,4-dihydroxy-3,3dimethylbutanoyl)amino]propanoic acid) and is eliminated from the human body (Xie et al., 2009).

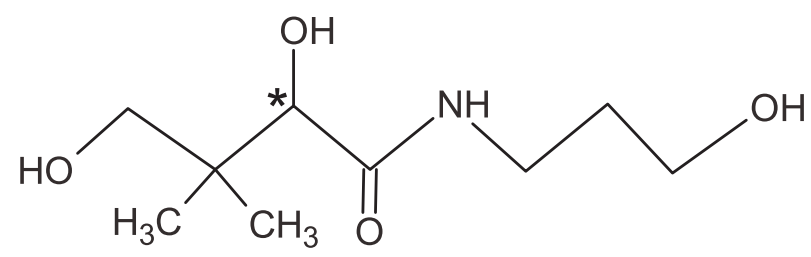

Fig. 1. Structure of panthenol.

For its moisturising and skin regeneration effect it is often added as constituent to cosmetics and personal-care products, like lotions and shampoo. It is also used in pharmaceuticals (skin products, nasal sprays, and eye drops), lozenges, dietary supplements. D-panthenol is used for the treatment of burns and minor skin injuries, helping to regenerate and improve the protective skin barrier and acceler- ate wound healing. It reduces inflammation and itching of skin (Camargo et al., 2011). In addition to its healing effects, panthenol can also cause allergic contact dermatitis (Chin et al., 2013). After application of a product containing panthenol, molecules of D-panthenol are converted to D-pantothenic acid which is a precursor of the biologically important coenzyme A. It plays an important role in the metabolism of carbohydrates, fats and nitrogen compounds (Płonka et al., 2012).

Separation of enantiomers is very important in the field of pharmaceutical analysis and the determination of optical purity of drugs and preparations. HPLC is the most frequently used technique for direct separation of enantiomers due to the availability of a large number of stationary phases containing different types of chiral selectors, high reproducibility, and universality. Selection of the chiral stationary phase (CSP) is an important step in the development of a chiral HPLC method. At present, the most commonly used CSPs are based on saccharides and macrocyclic antibiotic chiral selectors.

Several analytical methods such as supercritical fluid chromatography (Khater and West, 2015), gas chromatography, capillary electrophoresis (Kodama et al., 1998) and high performance liquid chromatography (HPLC) (Arai et al., 1989) have been used for panthenol enantiomers separation. HPLC offers several ways of enantiomeric separation of panthenol. Panthenol and its derivatives (3,5-dinitrophenyl carbamates; 3,5-dinitrobenzoyl esters) have been enantioseparated on ligand-exchange or 
Pirkle type chiral stationary phases (Zappala and Simpson, 1961; Song, 2003; Arai et al., 1989).

This work is focused on the development of a direct HPLC method for the separation and determination of panthenol enantiomers. Effect of the chiral stationary phase type and that of mobile phase composition on enantioseparation were studied. Two types of detectors, low-wavelength UV and polarimetric, were used. Suitable chromatographic conditions were applied for separation of enantiomers in samples of pharmaceutical preparations.

\section{Material and Methods}

\section{Chemicals}

D,L-panthenol (99\%), D-panthenol (99\%) were purchased from Thermo Fisher (Kandel) $\mathrm{GmbH}$ (part Alfa Aesar, Germany). Organic solvents for the preparation of mobile phases, methanol and acetonitrile, were purchased from VWR International (Slovakia), ethanol and hexane, were purchased from Merck (Slovakia) and propanol-2-ol from Thermo Fisher (Kandel) GmbH (Germany). All solvents were of HPLC gradient grade purity. Glacial acetic acid (100\% purity) and triethylamine (for synthesis) were purchased from Merck (Slovakia), acetic acid (99\% purity) was from Centralchem (Slovakia), trifluoroacetic acid (HPLC grade) was from Thermo Fisher (Kandel) GmbH (Germany). Panthenol containing pharmaceutical preparations, dietary supplement in form of capsules (Sample I) and tablets (Sample II) were obtained from a local pharmacy. Samples were stored in the original package in dark.

\section{Standard solutions}

Standard solutions of D,L-panthenol and D-panthenol were prepared in propanol-2-ol or ethanol (concentration of $10 \mathrm{mg} \mathrm{mL}^{-1}$ ) depending on the mobile phase type. Working standard solutions were prepared daily and stored at $4^{\circ} \mathrm{C}$.

\section{Sample preparation}

Five sample capsules/tablets were homogenized. An accurately weighed amount of approximately $0.9 \mathrm{~g}$ of sample I or $0.6 \mathrm{~g}$ of sample II was dissolved in $10 \mathrm{ml}$ of solvent (propanol-2-ol, ethanol); the mixture was sonicated in an ultrasonic bath $\left(25^{\circ} \mathrm{C}\right.$, $5 \mathrm{~min}$ ) and centrifuged (1500 rpm, $3 \mathrm{~min})$. The obtained supernatant was collected and diluted in the ratio of 1:3 with ethanol before injected onto the HPLC column. The extracts were stored at $8{ }^{\circ} \mathrm{C}$.

\section{HPLC instrumentation and conditions}

The HPLC system consisted of a delivery pump (Knauer, HPLG Pump 64), injection valve (Rheodyne, $20 \mu \mathrm{L}$ injection loop), thermostat (Incost
LCT 5100), and spectrophotometric (Knauer Variable Wavelength Monitor) and polarimetric (Chiralyser IBZ Messtechnik) detectors. Chromatographic datastation CSW 32 was used for data acquisition. Chiral chromatographic columns ChiraDex $(250 \mathrm{~mm} \times 4 \mathrm{~mm}, 5 \mu \mathrm{m}$; chiral selector was $\beta$-cyclodextrin), IP-CF6 $(250 \mathrm{~mm} \times 4 \mathrm{~mm}, 5 \mu \mathrm{m}$; chiral selector was isopropyl carbamate cyclofructan 6) and LUX ${ }^{\circledR}$ i-Amylose-1 $(250 \mathrm{~mm} \times 4.6 \mathrm{~mm}, 5 \mu \mathrm{m}$; chiral selector was amylose tris $(3,5$-dimethylphenylcarbamate)) were used for enantioseparation. The mobile phases consisted of hexane, propanol-2-ol or ethanol with an addition of ionic modifiers, trifluoroacetic acid and triethylamine. The flow rate was $0.8 \mathrm{~mL} \mathrm{~min} \mathrm{~m}^{-1}$ and the column temperature was $25^{\circ} \mathrm{C}$. The spectrophotometric detector was set to $210 \mathrm{~nm}$ and the polarimetric detector was operated at the measuring range of $0.8 \mathrm{mdeg} . / 10 \mathrm{mV}$ and the time constant of $0.4 \mathrm{sec}$

\section{Method validation}

The developed method was validated in terms of linearity, limit of detection (LOD), limit of quantification (LOQ), precision, and accuracy. Calibration curves were constructed after the injection of standard solutions with the concentrations from $1.0 \times 10^{-3}$ to $1.3 \mathrm{mg} \mathrm{mL}^{-1}$ and dependences of the mean peak area $(y)$ versus the corresponding concentration of the enantiomer $\left(\mathrm{x}, \mathrm{mg} \mathrm{mL}^{-1}\right)$ (nine concentration levels, $n=3$ ) were plotted. LOD and LOQ were expressed as signal-to-noise ratios of 3:1 and 10:1, respectively. They were determined by experimental measurement of a series of diluted solutions of DL- and D-panthenol. The recovery test was performed for samples spiked with DLpanthenol at three concentration levels. Spiked samples were maintained for 1 hour and then treated by the same procedure as unspiked samples. Seven independent extractions of each sample were carried out applying triplicate injections under the working conditions. Precision of the method was expressed as RSD \%.

\section{Results and Discussion}

This work was focused on the development of a direct HPLC method for the separation and determination of panthenol enantiomers. To find suitable chromatographic conditions, different types of chiral stationary phases and mobile phase compositions were tested.

\section{Separation of panthenol enantiomers} on $\beta$-cyclodextrin chiral stationary phase

Chiral selector $\beta$-cyclodextrin consists of seven glucopyranoside units linked with $\alpha$-1,4-bonds re- 
presented as toroids. Toroid shape of cyclodextrins contain larger and smaller openings exposing secondary and primary hydroxyl groups to the interactions with solvents. The inner cavity is hydrophobic because of the carbon-backbone of sugar moieties, while the surface of cyclodextrin is hydrophilic due to the presence of hydroxy groups. It allows to interact with analytes through different types of interactions (hydrogen bonds, dipole-dipole interactions, van der Waals interactions, $\pi-\pi$ interactions and inclusion in cavity). This type of CSP can be used in the normal phase (NP), reversed phase (RP), and polar-organic phase (PO) separation modes (Lämmerhofer, 2010; Vinodh et al., 2012).

The $\beta$-cyclodextrin based CSP was tested for enantioseparation of panthenol with hexane/ propanol-2-ol $(75 / 25, \mathrm{v} / \mathrm{v})$ as the mobile phase. These conditions were not suitable for panthenol enantiomers separation. Addition of ionic modifiers, trifluoroacetic acid $(0.2$ vol. \%) and triethylamine ( 0.1 vol. \% or 0.2 vol. \%) caused a decrease in the retention factor, however any resolution of the enantiomers was achieved. The $\beta$-cyclodextrin CSP in the NP separation mode was not suitable for enantio-recognition of panthenol probably due to the blocking of inner cavity of cyclodextrin by components of mobile phase and molecules of the analyte can interact only with polar surface (-hydroxyl groups) of cyclodextrin (Lämmerhofer, 2010).

\section{Separation of panthenol enantiomers on cyclofructan 6 based chiral stationary phase}

Cyclofructans are chiral selectors consisting of six or more D-fructofuranose units linked with $\beta$-(2-1) bonds. Each fructofuranose unit contains four stereogenic centers and three hydroxyl groups. As native cyclofructans have limited enantioselectivity in HPLC, usually, derivatized cyclofructans are used for enantioseparation. Derivatives with aliphatic or aromatic groups (isopropyl carbamate, $R$-naphthylethyl carbamate, and dimethylphenyl carbamate) were employed as chiral selectors. These CSPs can be used in all separation modes (NP, RP and PO), even though better separation of enantiomers was achieved in PO and NP separation modes (Cavazzini et al., 2011; Janečková et al., 2011; Sun and Armstrong, 2010).

CSP based on IP-CF6 and mobile phase consisting of hexane and popanol-2-ol were tested for enantioseparation of panthenol. The amount of propanol-2-ol in the mobile phase increased from 10 to 75 vol. \% and that of trifluoroacetic acid and triethylamine varied from 0.1 to 0.3 vol. $\%$. The increase of propanol-2-ol content led to increased values of selectivity factor and decreased values of retention factors (Tab. 1). Influence of the amount of ionic modifiers (acidic and/or basic) in the mobile phase hexane/propanol-2-ol (75/25, $\mathrm{v} / \mathrm{v}$ ) was also tested. It was observed that increasing the amount of acetic acid caused a decrease of the retention factor and a variation in the amount of triethylamine did not have any significant influence on the chromatographic characteristics. Partial separation of panthenol enantiomers with the resolution values from 0.51 to 0.85 (Tab. 1) was reached in mobile phase hexane/ propanol-2-ol/trifluoroacetic acid/triethylamine (75/25/0.2/0.1, v/v/v/v). Retention factor of the L-enantiomeric form of panthenol was lower

\section{Spectrophotometric detection $(210 \mathrm{~nm})$}

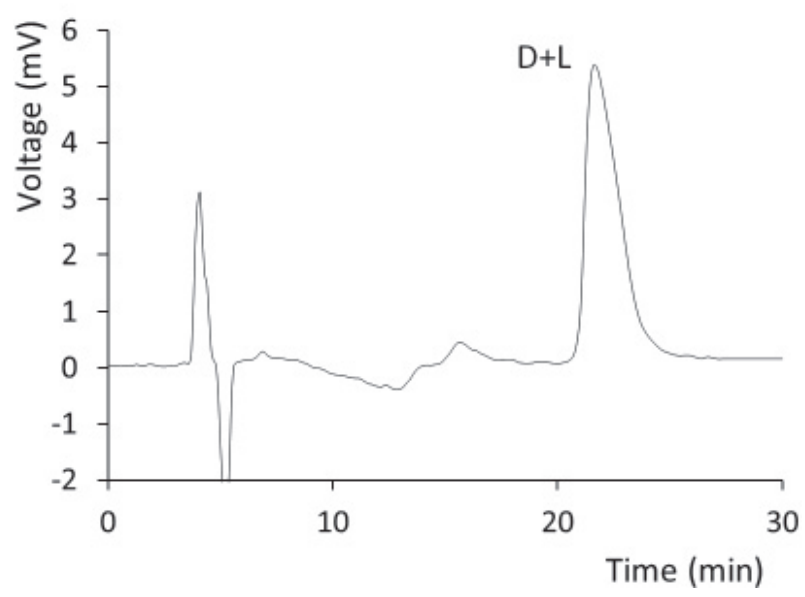

Polarimetric detection

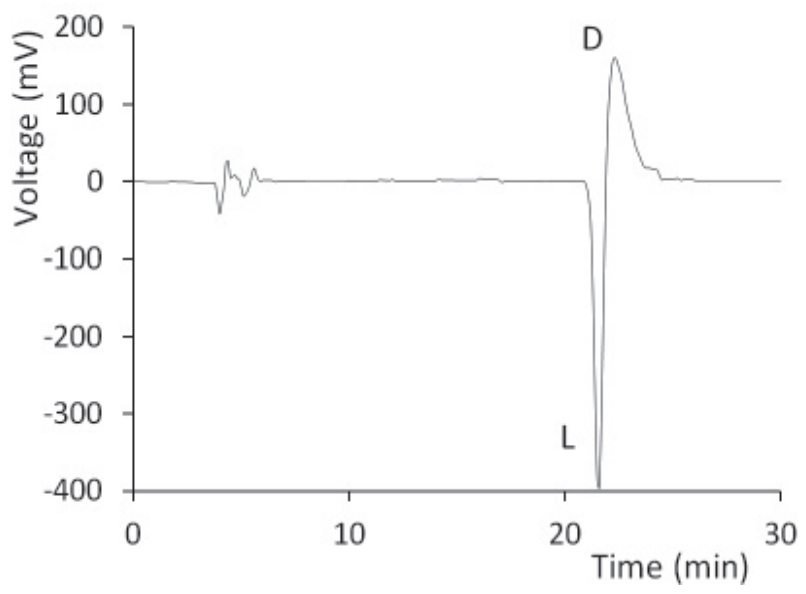

Fig. 2. Chromatograms of separation D,L-panthenol on isopropyl carbamate cyclofructan 6 CSP obtained with two types of detectors. Chromatographic conditions: mobile phase: hexane/propanol-2-ol/trifluoroacetic acid/triethylamine (75/25/0.2/0.1 v/v/v/v); column temperature: $25^{\circ} \mathrm{C}$; flow rate: $0.8 \mathrm{~mL} \mathrm{~min}^{-1}$. 
Tab. 1. Effect of mobile phase composition on retention factors $\left(k_{1}\right)$, resolution $\left(R_{\mathrm{s}}\right)$ and selectivity coefficient $(\alpha)$ of D,L-panthenol on different types of CSPs.

\begin{tabular}{|c|c|c|c|c|c|}
\hline Stationary phase & Mobile phase & & $k_{1}$ & $\alpha$ & $\boldsymbol{R}_{\mathrm{s}}$ \\
\hline \multirow[t]{7}{*}{ IP-CF6 ${ }^{1}$} & hexane/propanol-2-ol/ & $90 / 10 / 0.2 / 0.3$ & 17.82 & 1.04 & 0.81 \\
\hline & trifluoroacetic acid/ & $75 / 25 / 0.1 / 0.1$ & 3.73 & 1.04 & 0.72 \\
\hline & triethylamine & $50 / 50 / 0.1 / 0.1$ & 1.16 & 1.07 & 0.70 \\
\hline & & $25 / 75 / 0.1 / 0.1$ & 0.60 & 1.11 & 0.66 \\
\hline & & $75 / 25 / 0.2 / 0.2$ & 4.15 & 1.03 & 0.85 \\
\hline & & $75 / 25 / 0.1 / 0.2$ & 3.38 & 1.03 & 0.51 \\
\hline & & $75 / 25 / 0.2 / 0.1$ & 4.17 & 1.04 & 0.62 \\
\hline \multirow{9}{*}{$\begin{array}{l}\text { Amylose tris(3,5-dimethylphenyl- } \\
\text { carbamate })^{2}\end{array}$} & hexane/propanol-2-ol & $25 / 75$ & 0 & 1 & 0 \\
\hline & & $50 / 50$ & 0 & 1 & 0 \\
\hline & & $75 / 25$ & 0.57 & 1.14 & 0.71 \\
\hline & & $85 / 15$ & 1.49 & 1.19 & 1.12 \\
\hline & & $90 / 10$ & 4.15 & 1.23 & 1.61 \\
\hline & hexane/ethanol & $25 / 75$ & 0.15 & 1.63 & 1.11 \\
\hline & & $50 / 50$ & 0.29 & 1.78 & 2.13 \\
\hline & & $60 / 40$ & 0.38 & 1.77 & 2.49 \\
\hline & & $85 / 15$ & 3.07 & 1.05 & 0.47 \\
\hline
\end{tabular}

Chromatographic conditions: column temperature: $25^{\circ} \mathrm{C}$, ${ }^{1}$ polarimetric detection, ${ }^{2}$ spectrophotometric detection at $210 \mathrm{~nm}$; flow rate: $0.8 \mathrm{~mL} \mathrm{~min}^{-1}$.

than that of the D-enantiomeric form (Fig. 2). Aliphatic derivatization of hydroxyl groups in native cyclofructan 6 (IP-CF6), in comparison to the $\beta$-cyclodextrin chiral selector, disrupted the internal hydrogen bonding and made the molecule more "open". Carbamate groups can form interactions with amine groups of the analyte via dipolar interactions or hydrogen bonds (Cavazzini, 2011; Sun and Armstrong, 2010). Therefore, derivatized IP-CF6 CSP showed the capability to separate panthenol enantiomers.

\section{Separation of panthenol enantiomers on amylose based chiral stationary phase}

Amylose type chiral selector from the group of polysaccharides has stereoregular sequences of D-glucose units linked via $\alpha$-glycosidic bonds. In comparison with native amylose, its derivatives (esters and carbamates) were used as CSPs in HPLC due to their better enantioselectivity. CSP based on the amylose carbamate derivative used in this research contains $-\mathrm{NH}$ and $\mathrm{C}=\mathrm{O}$ functional groups which provide hydrogen bonds and dipol-dipol interactions with analytes. Increased enantioselectivity can be achieved using aromatic derivatized amylose CSPs providing $\pi-\pi$ interactions (Chen et al., 2007; Shen and Okamoto, 2015).

Many different mobile phases were evaluated on amylose tris(3,5-dimethylphenylcarbamate) CSP in the NP separation mode for panthenol enantiomers separation. Ethanol and propanol- 2-ol were used as organic modifiers (hexane/ propanol-2-ol or hexane/ethanol) in the mobile phase. Results of the mobile phase composition optimization are summarized in Table 1. Increase in the percentage of the alcohol modifier caused increased the enantiomers retention factors, selectivity coefficients and resolution values. The type of alcohol modifier has thus significant effect on the enantioresolution. Higher values of resolution and symmetrical peaks were obtained with mobile phases containing ethanol. As it is evident from Tab. 1 and Fig. 3, the optimal mobile phase for panthenol enantiomers separation was hexane/ethanol (60/40, v/v). Resolution reached the values of 2.49 within the analysis time below 10 min. Compared to conditions on IP-CF6 CSP, elution order of enantiomers was changed, D-enantiomer eluted with a lower retention factor than L-enantiomer.

Amylose type CSP shows the most efficient separation of panthenol enantiomers compared to the cyclodextrin and cyclofructan based CSPs under study. This is probably due to the presence of urethane groups in the chiral selector which prefer strong hydrogen bonds with enantiomers.

Application of HPLC method in real sample analysis Practical applicability of the developed HPLC method (optimal conditions: CSP based on amylose tris(3,5-dimethylphenylcarbamate) and mobile phase hexane/ethanol in the ratio of $60 / 40, \mathrm{v} / \mathrm{v}$ ) 

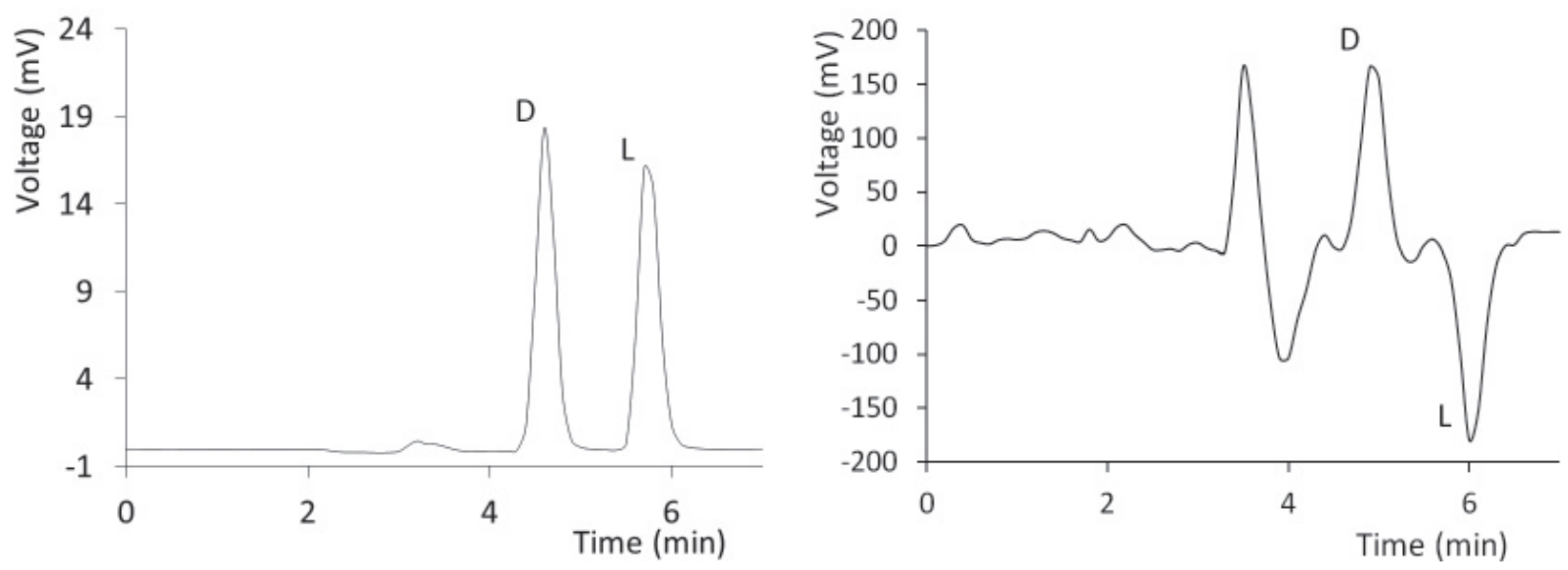

Fig. 3. Chromatograms of $\mathrm{D}, \mathrm{L}$-panthenol separation on amylose tris(3,5-dimethylphenylcarbamate) based CSP obtained with two types of detectors. Chromatographic conditions: mobile phase: hexane/ethanol $(60 / 40 \mathrm{v} / \mathrm{v})$; column temperature: $25^{\circ} \mathrm{C}$; flow rate: $0.8 \mathrm{~mL} \mathrm{~min}{ }^{-1}$.

Tab. 2. Analytical parameters for D- and L-panthenol determination by the HPLC-UV method.

\begin{tabular}{lcc}
\hline Parameter & D-panthenol & L-panthenol \\
\hline Linear concentration range $\left(\mathrm{mg} \mathrm{mL}^{-1}\right)$ & $1.0 \times 10^{-3}-1.3$ & $1.0 \times 10^{-3-1.3}$ \\
Regression equation & $A=325.9 c+2.3$ & $A=346.3 c+0.1$ \\
Coefficient of determination & 0.9987 & 0.9993 \\
Limit of detection $\left(\mathrm{mg} \mathrm{mL}^{-1}\right)$ & $0.3 \times 10^{-3}$ & $0.3 \times 10^{-3}$ \\
Limit of quantification $\left(\mathrm{mg} \mathrm{mL}^{-1}\right)$ & $1.0 \times 10^{-3}$ & $1.0 \times 10^{-3}$ \\
Repeatability $(\mathrm{RSD} \%)^{1}$ & 2.5 & 2.3 \\
\hline
\end{tabular}
${ }^{1} \mathrm{n}=7$.

Spectrophotometric detection (210 nm)

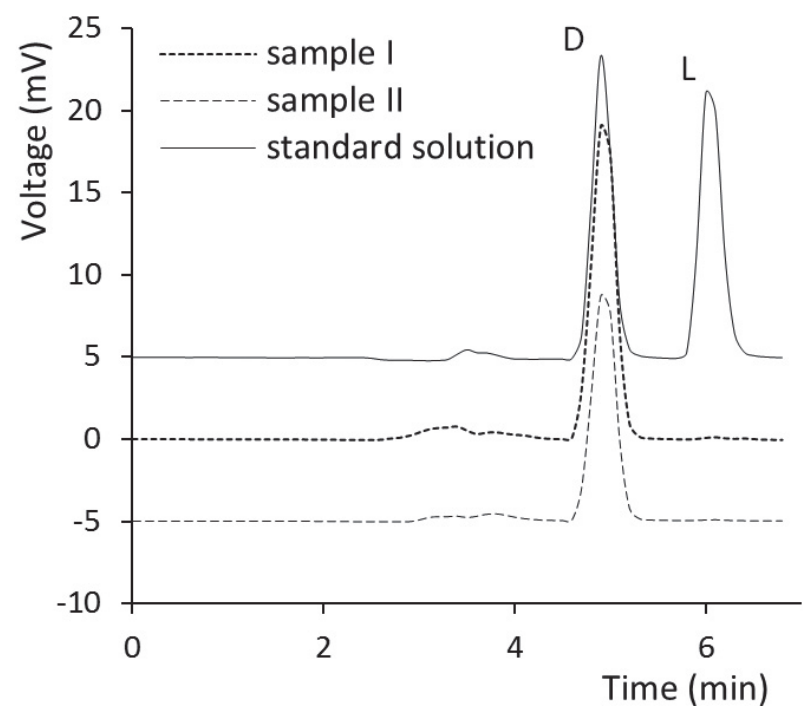

Polarimetric detection

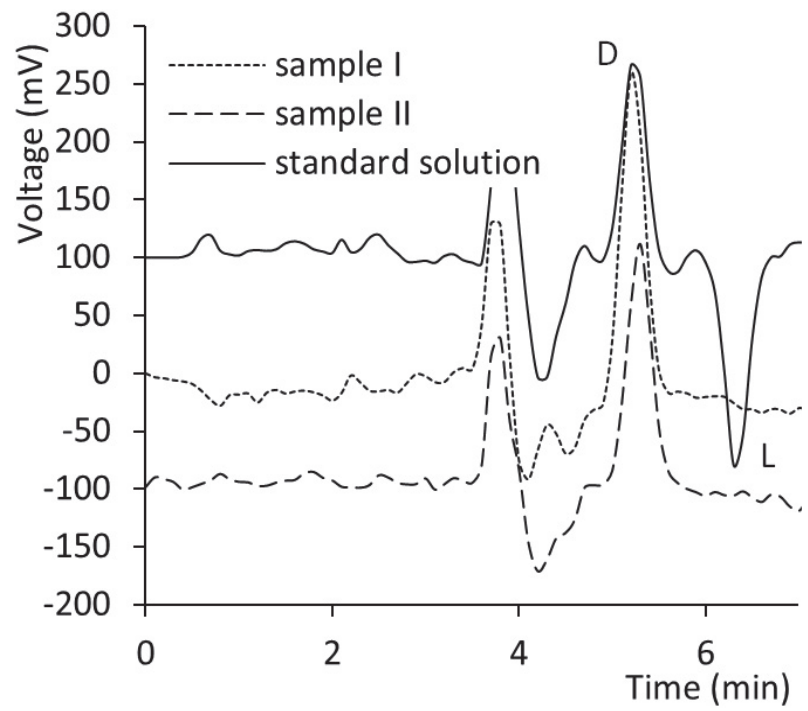

Fig. 4. Chromatograms of panthenol enantiomers separation in standard solution and pharmaceutical preparations. Chromatographic conditions: stationary phase: amylose tris(3,5-dimethylphenylcarbamate); mobile phase: hexane/ethanol $(60 / 40, \mathrm{v} / \mathrm{v})$, column temperature: $25^{\circ} \mathrm{C}$, flow rate: $0.8 \mathrm{~mL} \mathrm{~min}^{-1}$. 
was shown for the analysis of commercial pharmaceutical preparations. For the determination of panthenol enantiomers, the calibration curve method was used. Linear correlation between the average peak area (A) and the corresponding concentration of enantiomer (c, in $\mathrm{mg} \mathrm{mL}^{-1}$ ) was obtained in the concentration range from $1.0 \times 10^{-3}$ to $1.3 \mathrm{mg} \mathrm{mL}^{-1}\left(R^{2}>0.998\right)$. Analytical parameters are summarized in Tab. 2. The LOD and LOQ values were found to be $0.3 \times 10^{-3}$ and $1.0 \times 10^{-3} \mathrm{mg} \mathrm{mL}^{-1}$ for both enantiomers, respectively. Repeatability of the method was performed for a D,L-panthenol solution at the concentration level of $10.0 \mathrm{mg} \mathrm{mL}^{-1}$ with RSD values below $3 \%$ (Tab. 2). According to the manufacturer information, the tested pharmaceutical preparations contained D-panthenol. Chromatograms obtained by the proposed method employing spectrophotometric and polarimetric detection show that L-panthenol was also present in the tested samples (Fig. 4).

The amounts of enantiomers determined in sample I were: $22.2 \pm 2.0 \mathrm{mg} \mathrm{g}^{-1}(20.0 \pm 1.8 \mathrm{mg} /$ tablet $)$ for $\mathrm{D}$ panthenol, and $0.2 \pm 0.1 \mathrm{mg} \mathrm{g}^{-1}(0.2 \pm 0.1 \mathrm{mg} /$ tablet $)$ for L-panthenol. Enantiomeric composition of sample II was as follows: $106.0 \pm 5.7 \mathrm{mg} \mathrm{g}^{-1}(63.6 \pm 3.4 \mathrm{mg} /$ tablet) of D-panthenol, and $0.5 \pm 0.1 \mathrm{mg} \mathrm{\textrm {g } ^ { - 1 }}$ $(0.3 \pm 0.1 \mathrm{mg} /$ tablet $)$ of L-panthenol. Recovery tests were performed for spiked samples at three concentration levels: 5.0, 25.0, and $55.0 \mathrm{mg} \mathrm{g}^{-1}$ for sample 1; $8.0,28.0$, and $85.0 \mathrm{mg} \mathrm{g}^{-1}$ for sample II, reaching the values of above $92 \%$.

\section{Conclusion}

In this work, direct HPLG method for panthenol enantiomers separation and determination has been developed. Different types of CSPs based on cyclodextrin, derivatized cyclofructan 6 and amylose were tested in normal phase separation mode. From experimental data it can be concluded that the chiral selectors $\beta$-cyclodextrin and isopropyl carbamate cyclofructan 6 are not suitable for the separation of target enantiomers. The best enantioseparation was achieved on amylose tris(3,5-dimethylphenylcarbamate) CSP in the normal phase separation mode with mobile phase containing 60 vol. \% of ethanol in hexane. Analytical parameters obtained for the developed HPLC-UV method were as follows:
LOD $=0.3 \times 10^{-3} \mathrm{mg} \mathrm{mL} \mathrm{m}^{-1}$, linear concentration range of $1.0 \times 10^{-3}-1.3 \mathrm{mg} \mathrm{mL}^{-1}$, recovery above $92 \%$, and repeatability with RSD below $3 \%$ for both enantiomers. The developed method was used for the separation and determination of panthenol enantiomers in pharmaceutical preparations. It was found that both samples contain D-panthenol and a small amount of L-panthenol.

\section{Acknowledgements}

This study was funded by the Slovak Research and Development Agency under the contract no. APVV-15-0355 and STU Grant scheme for Support of Young Researchers and the project Excellent Teams of Young Researchers at STU.

\section{References}

Arai T, Matsuda H, Ōizumi H (1989) J. Chromatogr. 474: 405-410.

Armstrong DW, Tang Y, Chen S, Zhou Y, Bagwill C, Chen JR (1994) Anal. Chem. 66(9): 1473-1484.

Camargo FB, Gaspar LR, Campos PMBGM (2011) J. Cosmet. Sci. 62(4): 361-369.

Cavazzini A, Pasti L, Massi A, Marchetti N, Dondi F (2011) Anal. Chim. Acta 706(2): 205-222.

Chen X, Yamamoto C, Okamoto Y (2007) Pure Appl. Chem. 79(9): 1561-1573.

Chin MF, Hughes TM, Stone NM (2013) Contact Derm. 69(5): 321-322.

Deáková Z, Ďuračková Z, Lehotay (2014) J. Chem. Listy 108: 436-441.

Janečková L, Kalíková K, Vozka J, Armstrong DW, Bosáková Z, Tesařová E (2011) J. Sep. Sci. 34(19): 2639-2644.

Khater S, West C (2015) J. Pharm. Biomed. Anal. 102: 321-325.

Kodama S, Yamamoto A, Matsunaga A (1998) J. Chromatogr. A 811: 269-273.

Lämmerhofer M (2010) J. Chromatogr. A 1217(6): 814-856.

Płonka J, Toczek A, Tomczyk V (2012) Food Anal. Methods 5(5): 1167-1176.

Vinodh, M, Alipour FH, Mohamod AA, Al-Azemi TF (2012) Molecules 17(10): 11763-11799.

Shen J, Okamoto Y (2015) Chem. Rev. 116(3): 1094-1138.

Liu S (2003) Chin. J. Chromatogr. 21: 394-396.

Sun P, Armstrong DW (2010) J. Chromatogr. A 1217: 4904-4918.

Xie YM, Luo J, Tang XH, Yang D, Huo XF, Liu A, Song H (2009) Chromatographia 69(9-10): 1025-1029.

Zappala AF, Simpson CA (1961) J. Pharm. Sci. 50(10): $845-847$. 